\title{
Fabrication and Performance Evaluation of Air Pollutionremovable System with Fire Indicate for Fireworks Industry
}

\author{
*E. Ajithkumar, G. Shanmugasundar \\ Department of Mechanical Engineering, M.E. (Industrial Safety Engineering), Sri Sai Ram Institute of Technology, \\ Chennai, Tamil Nadu, India. \\ *Corresponding author Email: sitp20is03@sairamtap.edu.in
}

\begin{abstract}
Air pollution and aging populations are one of the most important issues facing in the development of all countries around the world. In this project work and effort has been made to develop a air pollution removable and fire indicated for fire work industry to safety purpose. The aim of this work is to develop a fire indicated and pollution removable that will use a collect fire work chemical powder around the working area. This model implies a conversion of rotary motion to the overall system. This motion is uses for chemical dust arrest by viscous liquid to all direction in the industry. In this model we can operate two pin switches for the clock wise and antilock wise direction. the gear motor is using to rotary motion. The rotary disk is mounted on the motor spindle. The tank is resting on the rotary disk. The exhaust fan is fitted in the top position of the tank. They are two way in the tank. One is inlet for suck the pollution air another one is outlet. Bottom of the tank dust dispatch valve attached. The fire indicates system is mounted on the rotary disk plate vertically. After this work performance test to the working area. This research provides a new perspective and the health effect of air pollution.
\end{abstract}

Keywords: Emergency Management, Risk, Incident, fireworks, air pollution, fire indicate.

\section{Introduction}

Air pollution is the contamination of air due to the presence of substances in the atmosphere that are harmful to the health of humans and other living beings, or cause damage to the climate or to materials. There are many different types of air pollutants, such as gases (including ammonia, carbon monoxide, sulfur dioxide, nitrous oxides, methane, carbon dioxide and chlorofluorocarbons particulates (both organic and inorganic), and biological molecules. Air pollution can cause diseases, allergies, and even death to humans; it can also cause harm to other living organisms such as animals and food crops, and may damage the natural environment (for example, climate change, ozone depletion or habitat degradation) or built environment (for example, acid rain). Both human activity and natural processes can generate air pollution. Air pollution is a significant risk factor for a number of pollution-related diseases, including respiratory infections, heart disease, COPD, stroke and lung cancer. Growing evidence suggests that air pollution exposure may be associated with reduced IQ scores, impaired cognition, increased risk for psychiatric disorders such as depression and detrimental per natal health. The human health effects of poor air quality are far reaching, but principally affect the body's respiratory system and the cardiovascular system. Individual reactions to air pollutants depend on the type of pollutant a person is exposed to, the degree of exposure, and the individual's health status and genetics. Outdoor air pollution alone causes 2.1 to 4.21 million deaths annually, making it one of the top contributors to human death. Overall, air pollution causes the deaths of around 7 million people worldwide each year, or a global mean loss of life expectancy (LLE) of 2.9 years, and is the world's largest single environmental health risk. Indoor air pollution and poor urban air quality are listed as two of the world's worst toxic pollution problems in the 2008 Blacksmith Institute World's Worst Polluted Places report. The scope of the air pollution crisis is enormous: $90 \%$ of the world's population breathes dirty air to some degree. Although the health consequences are extensive, the way the problem is handled is often haphazard. Productivity losses and degraded quality of life caused by air pollution are estimated to cost the world economy $\$ 5$ trillion per year but, along with health and mortality impacts, are an externality to the contemporary economic system and most human activity, albeit sometimes being moderately regulated and monitored. Various pollution control technologies and strategies are available to reduce air pollution. To reduce the impacts of air pollution, both international and national legislation and regulation have been implemented to regulate air pollution. Local laws, where well enforced, have led to strong public health improvements. At the international level, some of these efforts have been successful for example the Montreal Protocol was successful at reducing release of harmful ozone depleting chemicals or the 1985 Helsinki Protocol which reduced sulfur emissions, while other attempts have so far been less successful in implementation, such as international action on climate change. A fire alarm system warns people when smoke, fire, carbon monoxide or other fire-related emergencies are detected. These alarms may be activated automatically from smoke detectors and heat detectors or may also be activated via manual fire alarm activation devices such as manual call points or pull stations. Alarms can be either motorized bells or wall mountable sounders or horns. They can also be speaker strobes which sound an alarm, followed by a voice evacuation message which warns people inside the building not to use the elevators. 
Fire alarm sounders can be set to certain frequencies and different tones including low, medium and high, depending on the country and manufacturer of the device. Most fire alarm systems in Europe sound like a siren with alternating frequencies. Fire alarm electronic devices are known as horns in the United States and Canada, and can be either continuous or set to different codes. Fire alarm warning devices can also be set to different volume levels.

\section{Methodology}

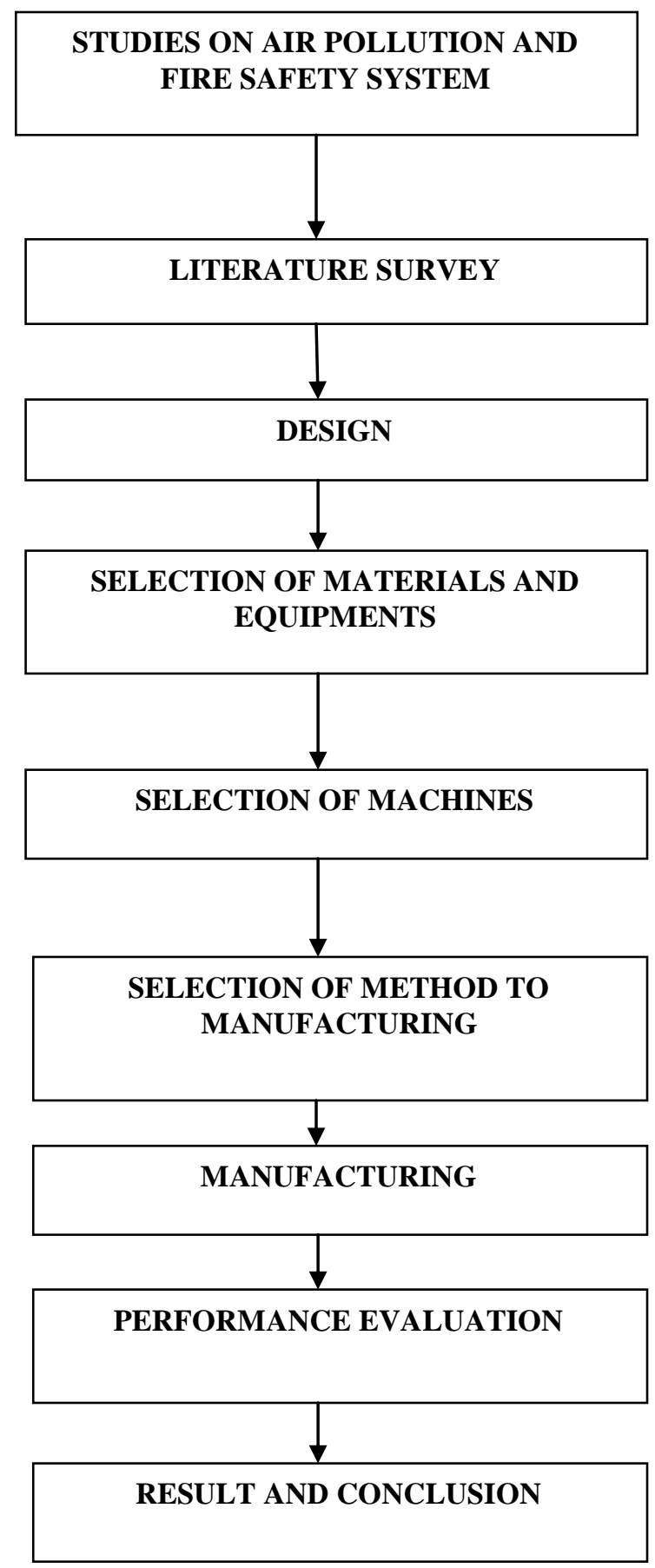

3. Material and Equipments

1. DC motor $-0.5 \mathrm{hp}$

2. Disk plate - MS Ø300mm

3. Tank - MS Ø250mm

4. Tank head - MS Ø250mm

5. Square plate $-\mathrm{MS} 300 \mathrm{~mm} \times 5 \mathrm{~mm}$

6. Inlet pipe - MS $\varnothing 50 \mathrm{~mm}$ 
7. Outlet pipe -MS Ø50mm

8. Exhaust Fan - 1500 rpm

9. Viscous liquid - gear oil $2 \mathrm{~L}$

10. Two pin switch

11. Stopper

12. Fire indicator

13. L angle plate - MS $32 \mathrm{~mm}$

14. Bolt nut $-10 \mathrm{~mm} 8$ nos

\section{Experimental Procedure}

The actual experimentation procedure is done as follows,

1. The raw material is prepared for base frame work using power hacksaw machine as per the required dimension.

2. L angle material is using to make the base frame work. After hacksaw cutting the material edges is grinding process using bench grinding machine.

3. The base frame material is arranging to required shape and dimension using try square. The try square using for check the parallel and perpendicular of material arrangement.

4. After arrangement the material is joining using arc welding technology as per the shape and dimension. After joining the material the base frame work is finished.

5. The motor is mounting on the base frame. before that drill the base material to fit the bolt nut for motor mounting work.

6. The rotary disk material is doing the turning and drilling process by lathe machine as the required dimension. the drilling process is suitable for motor shaft.

7. After finishing the rotary disk. it's fitting in the motor shaft.

8. The cylinder tank material to cut the required size using the power hacksaw machine. After the cutting process facing the edges by lathe machine using single point cutting tool.

9. The drilling operation doing in the cylinder tank for the inlet pipe fitting work and valve fitting work.

10. The gas cutting process doing in the square plate for fitting the exhaust fan. After the gas cutting the square plate is joining in the top of the cylinder tank and bottom of the cylinder tank head using arc welding technology

11. The drilling operation is doing in the square plate for exhaust fan fitting work. After drilling process joining the inlet pipe and valve using in the cylinder tank arc welding technology.

12. The tank head material is cutting the required dimension using power hacksaw machine and facing the edges of the tank head material.

13. Weld the square plate and cylinder plate in the tank head. After finishing this work drilling the tank head for the outlet pipe fitting work.

14. The outlet pipe is arranging for the joining the tank head.

15. The cylinder tank is mounting on the rotary disk by arc welding process. The fire indicator I also fitting on the rotary disk.

16. The L shape stopper is welding on the cylinder tank. The two pin switch in fitting on the base frame.

17. The exhaust fan is fitting on the cylinder tank square plate using bolt and but by spanners.

18. The viscous liquid is dropping in the cylinder tank and fitting o the cylinder tank head top of the tank using bolt nut by spanners.

19. The fire indicator system wiring work is doing in the product as per the required design. This system mounting in the rotary disk.

20. After finishing the all kind of joining work. Doing the alignment test using digital surface gages.

21. After the performance evaluation test doing the working area to check performance do the batter.

\section{Conclusion}

The air pollution removable system is made by mild steel material used as the base frame, cylinder tank, cylinder tank head, inlet pipe, outlet pipe, rotary disk etc. maximum the arc welding technology is using to make this system. Gas cutting process using to make exhaust fan holding plate. Some of the works using manufacturing technology like facing turning, drilling operation. The designed air pollution system using CAD technology to suitable shape and dimension. Before designing the system study the journals and articles area of the pollution control and fire safety hence, the air pollution removable system was fabricated with manufacturing technology and CAD technology. As the it is highly performance to compare other system for industrial safety, 


\section{References}

[1]. "World's Most Polluted Cities in 2020 - PM2.5 Ranking | AirVisual". www.iqair.com. Retrieved 1 February 2022.

[2]. Allen, J. L.; Klocke, C.; Morris-Schaffer, K.; Conrad, K.; Sobolewski, M.; Cory-Slechta, D. A. (June 2017). "Cognitive Effects of Air Pollution Exposures and Potential Mechanistic Underpinnings". Current Environmental Health Reports. 4 (2): 180-191. doi:10.1007/s40572-017-0134-3. PMC 5499513. PMID 28435996.

[3]. Jump up to: Ghosh, Rakesh; Causey, Kate; Burkart, Katrin; Wozniak, Sara; Cohen, Aaron; Brauer, Michael (28 September 2021). "Ambient and household PM2.5 pollution and adverse perinatal outcomes: A meta-regression and analysis of attributable global burden for 204 countries and territories". PLOS Medicine. 18 (9):e1003718. doi:10.1371/journal.pmed.1003718. ISSN 1549-1676. PMC 8478226. PMID 34582444.

[4]. Silva, Raquel A; West, J Jason; Zhang, Yuqiang; Anenberg, Susan C; Lamarque, Jean-François; Shindell, Drew T; Collins, William J; Dalsoren, Stig; Faluvegi, Greg; Folberth, Gerd; Horowitz, Larry W; Nagashima, Tatsuya; Naik, Vaishali; Rumbold, Steven; Skeie, Ragnhild; Sudo, Kengo; Takemura, Toshihiko; Bergmann, Daniel; CameronSmith, Philip; Cionni, Irene; Doherty, Ruth M; Eyring, Veronika; Josse, Beatrice; MacKenzie, I A; Plummer, David; Righi, Mattia; Stevenson, David S; Strode, Sarah; Szopa, Sophie; Zeng, Guang (2013). "Global premature mortality due to anthropogenic outdoor air pollution and the contribution of past climate change". Environmental Research Letters. 8 (3): 034005. Bibcode:2013ERL.....8c4005S. doi:10.1088/1748-9326/8/3/034005.

[5]. Chinnasamy, Sathiyaraj, M. Ramachandran, M. Amudha, and Kurinjimalar Ramu. "A Review on Hill Climbing Optimization Methodology." (2022).

[6]. Lelieveld, J.; Klingmüller, K.; Pozzer, A.; Burnett, R. T.; Haines, A.; Ramanathan, V. (25 March 2019). "Effects of fossil fuel and total anthropogenic emission removal on public health and climate". Proceedings of the National Academy of Sciences of the United States of America. 116 (15): 7192-7197. Bibcode:2019PNAS..116.7192L. doi:10.1073/pnas.1819989116. PMC 6462052. PMID 30910976. S2CID 85515425.

[7]. Jump up to:a b Lelieveld, Jos; Pozzer, Andrea; Pöschl, Ulrich; Fnais, Mohammed; Haines, Andy; Münzel, Thomas (1 September 2020). "Loss of life expectancy from air pollution compared to other risk factors: a worldwide perspective". Cardiovascular Research. 116 (11): 1910-1917. doi:10.1093/cvr/cvaa025. ISSN 0008-6363. PMC 7449554. PMID 32123898.

[8]. Venkateswaran, C., M. Ramachandran, Sathiyaraj Chinnasamy, Chinnasami Sivaji, and M. Amudha. "An Extensive Study on Gravitational Search Algorithm." (2022).

[9]. "Energy and Air Pollution" (PDF). Iea.org. Archived from the original (PDF) on 11 October 2019. Retrieved 12 March 2019.

[10]. "Study Links 6.5 Million Deaths Each Year to Air Pollution". The New York Times. 26 June 2016. Retrieved 27 June 2016.

[11]. Bharathi, Pon, M. Ramachandran, Kurinjimalar Ramu, and Sathiyaraj Chinnasamy. "A Study on Various Particle Swarm Optimization Techniques used in Current Scenario." (2022).

[12]. "Reports". WorstPolluted.org. Archived from the original on 11 August 2010. Retrieved 29 August 2010.

[13]. "Cheap air pollution monitors help plot your walk". European Investment Bank. Retrieved 18 May 2021.

[14]. "9 out of 10 people worldwide breathe polluted air, but more countries are taking action". www.who.int. Retrieved 18 May 2021.

[15]. "Assessing the risks to health from air pollution-European Environment Agency". www.eea.europa.eu. Retrieved 18 May 2021.

[16]. Chinnasami, S., M. Ramachandran, P. Vidhya, and M. Gowri. "Study of Evaluation Based on Distance from Average Solution on Moyamoya Disease and Energy application."

[17]. Jump up to:a b c World Bank; Institute for Health Metrics and Evaluation at University of Washington - Seattle (2016). The Cost of Air Pollution: Strengthening the Economic Case for Action (PDF). Washington, D.C.: The World Bank. xii.

[18]. Jump up to:a b c McCauley, Lauren (8 September 2016). "Making Case for Clean Air, World Bank Says Pollution Cost Global Economy \$5 Trillion". Common Dreams. Retrieved 3 February 2018.

[19]. Jump up to:a b "The Rising Cost of Smog". Fortune: 15. 1 February 2018. ISSN 0015-8259. 